\title{
ADV-MAC: Advertisement-based MAC Protocol for Wireless Sensor Networks
}

\author{
(Extended Abstract)
}

\author{
Surjya Ray, Ilker Demirkol and Wendi Heinzelman \\ Department of Electrical and Computer Engineering \\ University of Rochester \\ Rochester, New York \\ Email: \{ray, demirkol, wheinzel\}@ece.rochester.edu
}

\section{INTRODUCTION}

The major cause of energy waste in conventional MAC protocols such as IEEE 802.11 [1] is idle listening. To reduce this idle listening, Sensor-MAC (S-MAC) [2] used the concept of duty cycle. This reduces idle listening to a great extent. However, the duty cycle in S-MAC is fixed throughout the network operation. Thus, in case of low or no traffic, energy is still wasted in idle listening. Also, only one node can transmit in each frame within a neighborhood which reduces throughput in higher traffic loads.

Timeout-MAC (T-MAC) [3] tried to improve on S-MAC by introducing a variable duty cycle. It does so by introducing the idea of an activation period. An activation event can be any activity on the channel such as the firing of a periodic frame timer and reception of any data on the radio. Nodes will keep on renewing their timeout values and increase the activation period whenever an activation event occurs. When no activation event has occurred for a duration of a timeout period, the nodes go to sleep. The timeouts make the active period in T-MAC adaptive to variable traffic loads. Adaptive timeouts also enable more than one node to transmit in each frame in a neighborhood. Thus, T-MAC can handle variable traffic loads better than S-MAC. However, whenever an activation event occurs, all nodes that hear the event renew their TA timer even if they are not a part of the transmission. As a result, nodes still end up wasting valuable energy.

In this abstract, we present Advertisement MAC (ADV$\mathrm{MAC}$ ), an advertisement based MAC protocol for wireless sensor networks. Simulation results show that ADV-MAC provides a better way to conserve energy and at the same time to support variable traffic loads.

\section{ADV-MAC DESIGN OVERVIEW}

Fig. 1 illustrates the basic principles of ADV-MAC. The figure shows nodes A through $\mathrm{G}$, all of which are in transmission range of each other. Only the active times of the nodes are shown. In each frame, ADV-MAC has a fixed-length SYNC period and a fixed-length Advertisement period, followed by a variable-length data period and a variable-length sleep period.

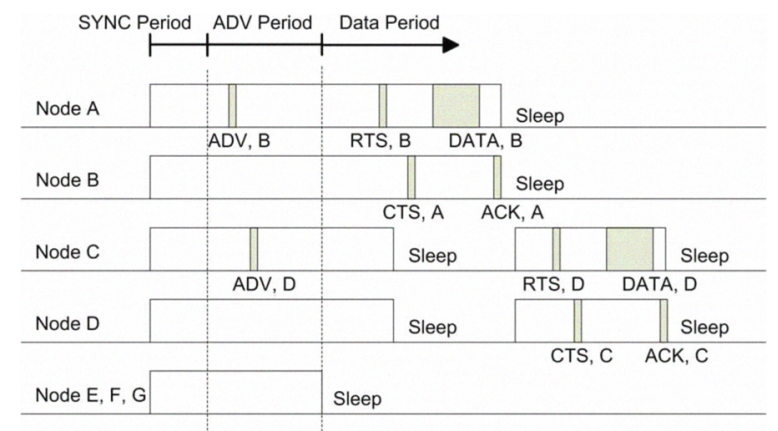

Fig. 1. Example of ADV-MAC protocol execution. The letters after the packets indicate the destination nodes.

It should be noted that while the data and sleep periods are variable, the total frame time is fixed.

The SYNC period is used for loose synchronization and is similar to that adopted by S-MAC [2]. The advertisement period is used to transmit ADV packets. If a node has any data to send, it will try to transmit an ADV packet during this period. ADV packets contain the ID of the intended receiver. If the ADV packet is received by its intended receiver, that node will be aware that there is data pending for it. Thus, after the end of the ADV period, only the nodes that sent ADV packets and the intended receivers that successfully received the ADV packets will be awake for the data time. The remaining nodes go to sleep. ADV packets do not require any reply back as RTS packets do, and thus transmitters will not know if ADV collision occurred. In case of an ADV collision, the nodes whose packets collided will not know of their collision and will be awake while their intended receivers will be asleep.

During the data period, nodes that have sent ADV packets will contend for the medium to send the data. Data exchange follows the exchange of RTS, CTS, data and ACK packets. Nodes go to sleep after overhearing an RTS or CTS destined for another node, which is called overhearing avoidance. The nodes whose ADV packets collided will also try to send RTS packet. However, their intended receivers will be asleep, and the sender nodes will eventually go to sleep after their CTS packets timeout. 


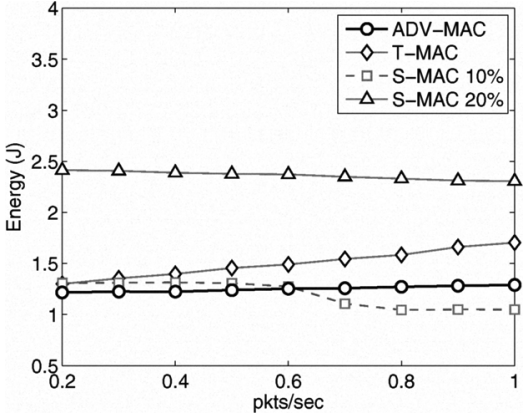

(a) Energy Consumption vs. Data Rate.

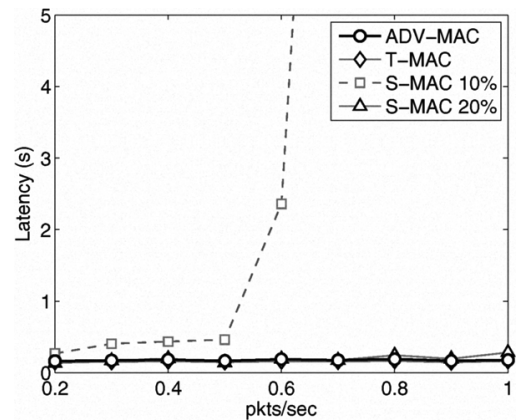

(b) Latency vs. Data Rate.

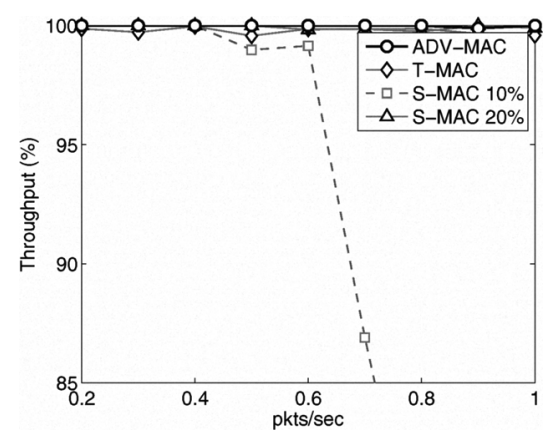

(c) Throughput vs. Data Rate.

Fig. 2. Performance comparison of ADV-MAC, T-MAC and S-MAC.

\section{EXPERIMENTS}

In our experiments, we compared the performance of the three protocols: S-MAC, T-MAC and ADV-MAC. We used energy consumption, throughput and latency as the three performance metrics for comparison. We performed all simulations in ns 2.29 [4]. We use two different duty cycle settings (10\% and $20 \%$ ) for S-MAC, because one fixed duty cycle is not suitable for all traffic loads investigated. The frame time for $10 \%$ duty cycle is $238.4 \mathrm{~ms}$, and we set this frame time for T-MAC and ADV-MAC as well. We used a duration of $15 \mathrm{~ms}$ for the time-out periods of T-MAC as in [3] as well as for the Advertisement period of ADV-MAC. Since transmit, receive and idle listening have close energy consumption values [5][6], we set a common value of $55.8 \mathrm{~mW}$ for all three operations in accordance with [5]. The transmission rate is $250 \mathrm{Kbps}$, and the transmission range is $100 \mathrm{~m}$, while the interference, i.e., carrier sense range, is $200 \mathrm{~m}$. All nodes in the simulations are placed randomly. We use T-MAC with over-hearing avoidance, as it is used in the simulations in [3]. In the preliminary investigations, we investigated the effects of traffic load on energy consumption, latency and throughput. We consider an area of $50 \mathrm{~m} \times 50 \mathrm{~m}$ with all nodes in transmission range of each other. There are 20 nodes in the area including 5 sources. The traffic load is varied by increasing the data rate from 0.2 packet/second to 1 packet/second.

Fig. 2(a) shows the energy consumptions obtained from the simulations. Figs. 2(b) and 2(c) show the corresponding latency and throughput values, respectively. As seen from the figure, ADV-MAC and S-MAC with $10 \%$ duty cycle give the best energy consumption results. However, as seen from the corresponding latency and throughput values, S-MAC with such a low duty cycle actually cannot handle the high traffic loads and gives very poor throughput and very high latency results. However, ADV-MAC presents stable latency and throughput results for all traffic loads, showing its resiliency to variable data traffic loads and high traffic loads. As data rate increases beyond 0.5 packets/second, $10 \% \mathrm{~S}-\mathrm{MAC}$ is no longer sufficient because of high latency and low throughput. At high data rates, $20 \%$ duty cycle gives acceptable values of latency and throughput for S-MAC. However, the energy consumption of ADV-MAC is $44 \%$ less than the energy consumption of S-
MAC with $20 \%$ duty cycle at the highest data rate. It is seen that as data rate increases, the energy consumption of ADVMAC increases very little, but that of T-MAC increases much faster. This happens because nodes that are not a part of a data exchange can selectively go to sleep in ADV-MAC, but all nodes in the carrier sense range must be awake in T-MAC. From Fig. 2(a) we can see that the energy consumption of ADV-MAC at higher data rates is up to $24 \%$ less than that of TMAC. ADV-MAC also has the least latency and the maximum throughput for all data rates. S-MAC with $20 \%$ duty cycle and T-MAC also have the least latency and maximum throughput, but their energy consumptions are much higher than ADVMAC, as pointed out before. Thus ADV-MAC successfully adapts to traffic load, providing minimum energy consumption with maximum throughput and minimum latency over all traffic conditions.

\section{ONGOING AND FUTURE WORK}

Currently we are doing detailed performance evaluation of ADV-MAC in single-hop as well as multihop scenarios. In the future, we plan to optimize the lengths of the ADV period and the contention window. Updates on this ongoing work will be posted on our lab's web site [7].

\section{ACKNOWLEDGMENT}

This work was supported in part by NSF CAREER grant \#CNS-0448046 and in part by a Young Investigator grant from the Office of Naval Research, \#N00014-05-1-0626.

\section{REFERENCES}

[1] "IEEE Standard 802.11, Wireless LAN Medium Access Control (MAC) and Physical Layer (PHY) Specifications," LAN MAN Standards Committee of the IEEE Computer Society., June 1999.

[2] W. Ye, J. Heidemann, and D. Estrin, "An energy-efficient mac protocol for wireless sensor networks," in Proceedings of the IEEE Infocom. New York, NY, USA: IEEE, June 2002, pp. 1567-1576.

[3] T. van Dam and K. Langendoen, "An adaptive energy-efficient mac protocol for wireless sensor networks," in SenSys '03: Proceedings of the 1st international conference on Embedded networked sensor systems. New York, NY, USA: ACM, 2003, pp. 171-180.

[4] "The Network Simulator NS-2." [Online]. Available: http://www.isi.edu/nsnam/ns/

[5] "Crossbow Technology Inc." [Online]. Available: http://www.xbow.com/

[6] "Sentilla Corporation." [Online]. Available: http://www.sentilla.com/

[7] "Wireless Communications and Networking Group." [Online]. Available: http://www.ece.rochester.edu/research/wcng/ 